A. K. Dupree and A. O. Benz, Eds.

\title{
An Observational Test for Coronal Heating Models
}

\author{
Lidia van Driel-Gesztelyi ${ }^{1,2,3}$
}

1. Mullard Space Science Laboratory, University College London, Holmbury St. Mary, Dorking, Surrey, RH5 6NT, UK

2. Konkoly Observatory, H-1525 Budapest, P.O. Box 67, Hungary

Pascal Démoulin

3. Observatoire de Paris, LESIA, FRE 2461 (CNRS), F-92195 Meudon Cedex, France

Cristina H. Mandrini

Instituto de Astronomía i Física del Espacio, IAFE, CC. 67, Suc. 28, 1428 Buenos Aires, Argentina

Louise K. Harra

Mullard Space Science Laboratory, University College London, Holmbury St. Mary, Dorking, Surrey, RH5 6NT, UK

James A. Klimchuk

Naval Research Laboratory, Code 7675, Washington, DC 20375-5352, USA

\begin{abstract}
We correlate the evolution of the mean X-ray flux, emission measure and temperature (Yohkoh SXT \& BCS) with the magnetic flux density (SOHO/MDI) in active region NOAA 7978 from its birth throughout its decay, for five solar rotations. We show that these plasma parameters together with other quantities deduced from them, such as the density and the pressure, follow power-law relationships with the mean magnetic flux density $(\bar{B})$. We derive the dependence of the mean coronal heating rate on the magnetic flux density. We use the obtained scaling laws of coronal loops in thermal equilibrium to derive observational estimates of the scaling of the coronal heating with $\bar{B}$. These results are used to test the validity of coronal heating models. We find that models based on the dissipation of stressed, current-carrying magnetic fields are in better agreement with the observations than models that attribute coronal heating to the dissipation of MHD waves injected at the base of the corona. This confirms, with smaller error bars, previous results obtained for individual coronal loops, as well as for the global coronal emission of the Sun and cool stars.
\end{abstract}



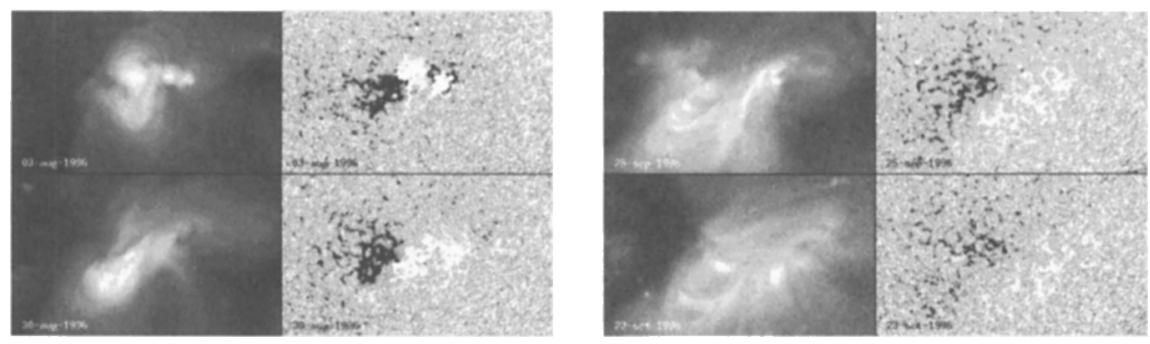

Figure 1. Long-term evolution of the soft Xray emission (Yohkoh/SXT) and photospheric longitudinal magnetic field (SoHO/MDI) for AR 7978. The evolution from the second to the fifth rotations is shown here at central meridian passages.
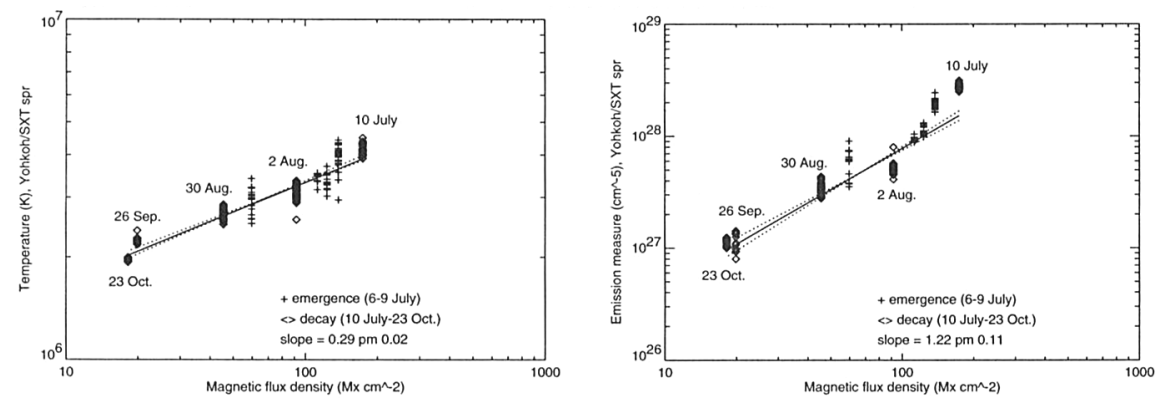

Figure 2. Log-log plots of the temperature and emission measure (Yohkoh/SXT) versus magnetic flux density $(\bar{B})$. The solid line shows the linear least-squares fit and the two dashed lines correspond to the $3 \sigma$ error in the slope. Only the decaying phase (points marked with $\diamond$ ) are included in the least-squares fit. The same analysis has been done for Yohkoh/BCS data.

\section{Introduction}

The solar corona, at a temperature of above $10^{6} \mathrm{~K}$, originates from an important energy supply to the atmosphere. This energy input increases both the temperature and the density of the plasma by orders of magnitude. Most of the present coronal heating models propose that this energy has a magnetic origin; however, no consensus has yet been reached about the physical mechanism by which the magnetic energy is converted into heat. To make further progress, it is important to establish how these physical quantities, whose variations are the consequence of the energy input, relate to the magnetic field.

\section{Scaling laws between coronal plasma parameters and magnetic flux density}

We analyze the evolution of plasma parameters as a function of magnetic flux density in active region NOAA 7978 from its birth throughout its decay (Fig. 1). We use SoHO/MDI data to derive magnetic observables, as well as Yohkoh/SXT 


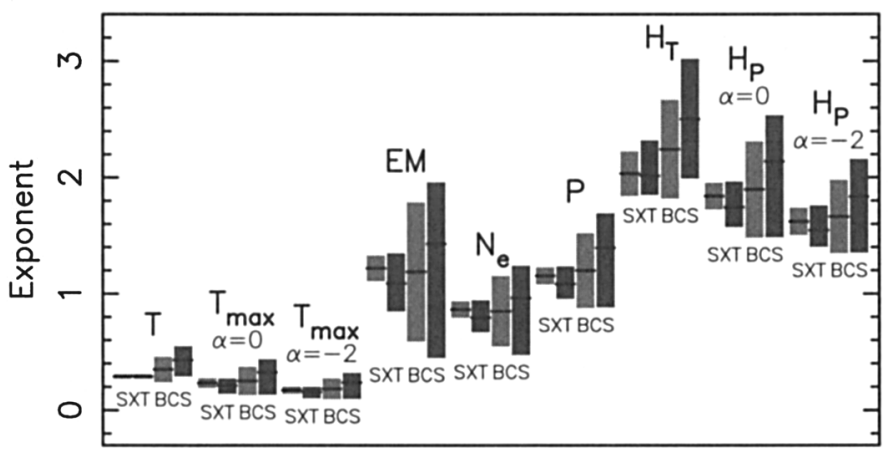

Figure 3. Ranges for the exponents found for the mean physical parameters in the scaling law equations: parameter $\propto \bar{B}^{\text {exponent }}(\bar{B}$ : magnetic flux density). $H_{T}$ and $H_{P}$ are the heating rate derived from the observations and the thermal model. For $T_{\mathrm{m}} \mathrm{x}$ and $H_{P}$ two values of $\alpha$ are shown (coronal radiative losses $\propto T^{\alpha}$ ). The $\pm 3 \sigma$ error range of a normally distributed statistics is light shaded. The $90 \%$ confidence interval of the non-parametric statistics is dark shaded.

and Yohkoh/BCS data to determine the global evolution of the temperature and the emission measure of the coronal plasma at times when no significant brightenings were observed. We find that the mean X-ray flux and derived parameters, temperature, emission measure, density and the pressure of the plasma in the AR follow power-law relationships with the mean magnetic flux density $(\bar{B})$ (Fig. 2). The exponents $(b)$ of these power-law functions $\left(a \bar{B}^{b}\right)$ are derived using two different statistical methods, a classical least-squares method in log-log plots and a non-parametric method, which takes into account the fact that errors in the data may not be normally distributed. Both methods give similar exponents, within error bars, for the mean temperature and for both instruments (Fig. 3).

As a next step, we derive the dependence of the mean coronal heating rate on the magnetic flux density. We use the scaling laws of coronal loops in thermal equilibrium to derive four observational estimates of the scaling of the coronal heating with $\bar{B}$ (two from SXT and two from BCS observations; see Fig. 3). These results are used to test the validity of coronal heating models.

\section{Testing coronal heating models}

Most coronal heating models give a heating rate per unit volume which can be expressed in the following generic way:

$$
H_{m} \propto B L^{b} N_{e}^{c} V^{d} R^{e}
$$

where $B$ is the coronal field strength, $L$ is the loop length, $N_{e}$ is electron density, $V$ is the transverse (horizontal) velocity at the base of the corona, and $R$ is 

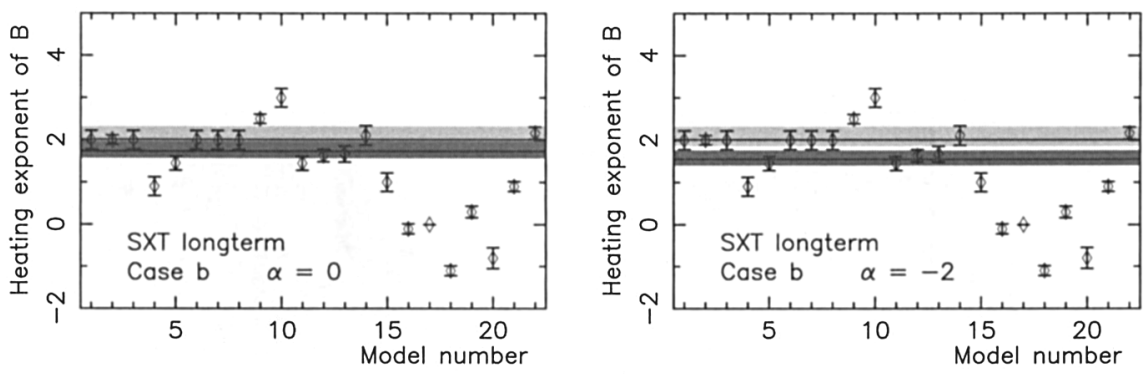

Figure 4. Comparison of the heating rate, $\langle H\rangle$, versus magnetic flux density, $\bar{B}$, scaling law, $<H>\propto \bar{B}^{\widehat{H}}$, as deduced from observations and models. We show the case for $\alpha$ (exponent for the coronal radiative losses $)=0$ and $=-2$. The horizontal lines are the two exponents $(\widehat{H T}$ - lighter grey, $\widehat{H P}$ - darker grey) as deduced from our observations. The plotted points with error bars indicate the power-law index $\widehat{H m}$ predicted by the models (cf. Table 1).

the loop radius or the transverse scale length for the magnetic or flow field, depending on the model. The coefficients $a$ through $e$ predicted by a variety of models (Table 1) are given in Mandrini et al., 2000.

As a proxy of the coronal field $B$ we use the photospheric flux density $\bar{B}$. If we know how $L, N_{e}, V$, and $R$ depend on $\bar{B}$, then the general equation can be written as:

$$
H_{m} \propto \bar{B}^{\widehat{H m}}
$$

where the exponent $\widehat{H m}$ is model dependent. The comparison of $\widehat{H m}$ with the exponents $\widehat{H P}$ and $\widehat{H T}$, deduced from our observations, lets us test the various models (Fig. 3).

The dependence of $L$ on $\bar{B}$ is expressed by $L \propto \sqrt{A} \propto \bar{B}^{\widehat{A} / 2}(A$ is the AR area). The $N_{e}$ dependence has been determined from observations by us. This leaves $V$ and $R$, for which we adopt two cases. Case (a): the coronal and photospheric quantities are identical, while Case (b) takes into account that the photospheric field is strongly concentrated in thin flux tubes.

\section{Conclusions}

Values derived from SXT and BCS observations for exponents $\widehat{H P}$ and $\widehat{H T}$ are very close to 2 (Fig. 3 ). This implies that the heating rate per unit volume is simply proportional to the coronal magnetic energy density when it is expressed only in function of the magnetic field strength !

Taking into account the observed fact that the photospheric field is concentrated in thin flux tubes [Case (b)], both the SXT and BCS results are compatible with three groups of models (Fig. 4): models considering a stochastic build up of energy (1-3), models including current layers (6-8) and models implying MHD turbulence (11-14 and 22). This result agrees with the independent result of Schrijver \& Aschwanden (2002) that constrains the heating mechanisms by 
Table 1. Models tested (see full references in Mandrini et al., 2000)

\begin{tabular}{|c|c|c|}
\hline \multirow{2}{*}{\multicolumn{3}{|c|}{$\begin{array}{l}\text { No. Physics involved } \\
\text { Stressing models }\end{array}$}} \\
\hline & & \\
\hline $1-3$ & Stochastic Buildup & $\begin{array}{l}\text { Surrock \& Uchida (1981); Berger (1991); } \\
\text { Parker (1988); Berger (1993); }\end{array}$ \\
\hline & Reconnection & $\begin{array}{l}\text { Galsgaard \& Nordlund (1997) } \\
\text { Parker (1983) }\end{array}$ \\
\hline $6-8$ & Current Layers & $\begin{array}{l}\text { van Ballegooijen (1986); Hendrix et al. (1996); } \\
\text { Galsgaard \& Nordlund (1996) }\end{array}$ \\
\hline 9 & Current Sheets & Ali \& Amari (1997) \\
\hline 10 & Taylor Relaxation & $\begin{array}{l}\text { Heyvaerts \& Priest (1984); Vekstein et al. (1993) } \\
\text { Browning \& Priest (1986) }\end{array}$ \\
\hline $11-14$ & Turbulence & $\begin{array}{l}\text { Einaudi et al. (1996); Dmitruk \& Gómez (1997) } \\
\text { Heyvaerts \& Priest (1992); Inverarity et al. (1995); } \\
\text { Inverarity \& Priest (1995a); Milano et al. (1997) }\end{array}$ \\
\hline \multicolumn{3}{|c|}{$\begin{array}{l}\text { Wave models } \\
15\end{array}$} \\
\hline 15 & Resonance & Hollweg (1985) \\
\hline $17-20$ & Resonant Absorption & $\begin{array}{l}\text { Ofman et al. (1995); Ruderman et al. (1997); } \\
\text { Halberstadt \& Goedbloed (1995) }\end{array}$ \\
\hline 21 & Current Layers & Galsgaard \& Nordlund (1996) \\
\hline 22 & Turbulence & Inverarity \& Priest (1995b) \\
\hline
\end{tabular}

comparing the modeling of the X-ray emission of the Sun and cool stars to observations. More details on this project can be found in van Driel-Gesztelyi et al. (2003) and Démoulin et al. (2003).

Acknowledgments. The authors thank the SOHO/MDI consortium for the SOHO data and the MSSL UCL Solar UK Research Facility for Yohkoh data. SOHO is a joint project by ESA and NASA. P.D. and C.H.M. acknowledge financial support from ECOS (France) and SECyT (Argentina) through their cooperative science program (A01U04). L.v.D.-G. acknowledges the Hungarian government grant OTKA T-038013. The work of J.A.K. was supported by NASA and the Office of Naval Research. P.D., L.v.D.-G., and L.K.H. acknowledge travel support from the Royal Society Joint Project (MSSL/UCL Observatoire de Paris, Meudon). L.K.H. is grateful to PPARC for the award of an advanced fellowship.

\section{References}

Démoulin, P., van Driel-Gesztelyi, L., Mandrini, C. H., Klimchuk, J.A. \& Harra, L. 2003, ApJ, 586, 592

Mandrini, C.H., Démoulin, P., \& Klimchuk, J.A. 2000, ApJ, 530, 999

Schrijver, C. J., \& Aschwanden, M. J. 2002, ApJ, 566, 1147

van Driel-Gesztelyi, L., Démoulin, P., Mandrini, C. H., Harra, L., \& Klimchuk, J.A. 2003, ApJ, 586, 579 